\title{
MONITORING OF MOVEMENT BEHAVIORS OF CHIRONOMID LARVAE AFTER EXPOSURE TO DIAZINON USING FRACTAL DIMENSION AND SELF-ORGANIZING MAP
}

\author{
C.W. JI ${ }^{1}$, S.H. LEE ${ }^{2,3}$, K.-H. CHOI ${ }^{1}$, I.-S. KWAK ${ }^{4}$, S.G. LEE ${ }^{5}$, E.Y. CHA ${ }^{5}$, S.-K. LEE ${ }^{6}$ \& T.-S. CHON ${ }^{1}$ \\ ${ }^{1}$ Division of Biological Sciences, Pusan National University, Republic of Korea. \\ ${ }^{2}$ Department of Physics, Pusan National University, Republic of Korea. \\ ${ }^{3}$ Department of Entomology and Nematology, University of Florida, Ft. Lauderdale, Florida, USA. \\ ${ }^{4}$ Faculty of Marine Technology, Chonnam National University, Republic of Korea. \\ ${ }^{5}$ Department of Electronics and Engineering, Pusan National University, Republic of Korea. \\ ${ }^{6}$ Toxicology Research Center, Korea Research Institute of Chemical Technology, Republic of Korea.
}

\section{ABSTRACT}

Response behaviors of chironomid larvae, Chironomus riparius, were analyzed by fractal dimension and artificial neural networks after the specimens were exposed to an insecticide. The two-dimensional movement tracks of individual specimens were continuously recorded before and after the treatments of diazinon at the concentration of $0.001 \mathrm{ppm}$. Fractal dimensions were obtained from the movement tracks and appeared to decrease consistently after the treatments. Subsequently, parameters such as speed, acceleration, stop number, maximum length, locomotory rate, and meander were extracted from the 1-h segments and were used as input data for training with the self-organizing map (SOM). The movement tracks were accordingly classified before and after the treatments by the trained SOM. Fractal dimension and the SOM were useful for extracting information residing in behavioral data and could be an alternative tool for automatically detecting toxic substances in an aquatic environment.

Keywords: chironomids, diazinon, fractal dimension, monitoring, movement, pesticide, response behavior, selforganized map.

\section{INTRODUCTION}

Recently, behavioral detection of indicator species has been regarded as an efficient monitoring tool in aquatic ecosystems. Response behaviors have been reported to be sensitive to sub-lethal exposures to various chemical pollutants [1,2]. Dutta et al. [2] studied the relationships between brain acetylcholine-esterase activity and optomotor behaviors of bluegills in different concentrations of diazinon, and indicated that behavioral bioassay may be more sensitive than other types of testing. Blüebaum-gronau et al. [3] characterized movement behaviors such as rheotaxis disorientation when affected by toxic substances. Fishes were frequently used for monitoring the presence of toxic chemicals [4-8]. Oshima et al. [9] observed the suppression of sexual behavior in male medaka exposed to estradiol. Recently, behavioral monitoring in response to various toxic chemicals has been conducted on aquatic invertebrates: crustaceans [10-12], crustacean parasite [13], snails [14], and insects [15].

Previous studies on behavioral response, however, have been mainly focused on the analyses of single behaviors or combination of single behaviors. Computational analyses on overall movement have not been extensively carried out due to complexity residing in behavioral data. To cope with the problem of complexity in the data, theoretical research has been conducted on analyzing the movement tracks along with the development of study on biological motion [16]. Alt [16] modeled the movement of organisms, such as the circling paths of gametes or the meander searches by isopods, while Scharstein [17] revealed a complex directional autocorrelation function regarding walking paths of beetles in the absence of orienting stimuli. Recently, exploration behaviors of rats were studied on dynamic perspectives [18, 19] and statistical discrimination of motion [20]. Gerhardt et al. [15, 21, 22] used the quadrupole impedance conversion technique to monitor stress from chemical disturbance on various aquatic invertebrates including Daphnia, Gammarus, and mayflies. 
Among the computational studies on evaluating the movement data, measure of the movement tracks has been efficiently used to quantitatively characterize behavioral states of the specimens. Tourtellot et al. [23] analyzed the move length and turn definition in the analysis of orientation of cockroaches. Recently, fractal dimension [24] has been used for addressing behavioral changes with mathematical precision and ease of comprehension. Johnson et al. [25] and Wiens et al. [26, 27] suggested that fractal dimensions of pathways provide information on movement characterization not available through absolute measures of pathway configurations. Alados et al. [28] reported that fractal dimension declined with stress from pregnancy and parasitic infection.

Although the computational methods were effective in revealing data structure, the parameters (fractal dimension) obtained from mathematical analyses are highly compressed and usually express behavioral states of the specimens only in overall terms. Local and global changes in the movement data, however, are both important in characterizing the tracks' shape and are equally critical in detecting behavioral changes. Recently, artificial neural networks have been used for recognizing response behaviors. Kwak et al. [8] utilized the multi-layer perceptron (MLP) and detected changes in the movement patterns of medaka fish before and after the treatments of diazinon. The MLP, however, recognized the movement patterns in the supervised manner by requiring the templates $a$ priori. The self-organizing map (SOM), on the other hand, is useful for learning the pattern in an unsupervised manner, and has been implemented to classify response behaviors of indicator organisms such as cockroaches [29] and medaka fishes [30] without previous knowledge.

Recently, fractal dimension and artificial neural networks have been used in combination to extract the local and global information at the same time. Ji et al. [31] applied the MLP and fractal dimension to medaka fish treated with copper. The movement patterns were initially chosen by experienced observers. Subsequently, the parameters were selected from the movement patterns and were trained with the MLP. After recognition of the patterns, fractal dimension was used to confirm changes in data structure before and after the treatments. In this study, however, we intended to analyze the movement data without prior knowledge. Fractal dimension was first used to reveal overall changes in data structure after the treatments, while the SOM was subsequently implemented to classify movement patterns.

\section{MATERIALS AND METHODS}

2.1 Test specimens and observation

A strain of Chironomus riparius provided by the Korea Research Institute of Chemical Technology (KRICT; Taejeon, Korea) was used for observation. The stock population was reared with an artificial dry diet (Tetramin) under the light regime of L10:D14 (light phase from 8:00 a.m. to 6:00 p.m.) at a temperature of $23 \pm 0.5^{\circ} \mathrm{C}$. Fourth instar larvae were individually placed in an observation aquarium $(6.5 \mathrm{~cm} \times 5.5 \mathrm{~cm} \times 1.5 \mathrm{~cm})$. The height of the aquarium was minimized in such a manner that twodimensional image of the specimens was accordingly produced while the height did not constrain swimming of the specimens.

Diazinon, dissolved in dimethylsulfoxide (DMSO; $10 \mathrm{mg} / \mathrm{L}$ ), was introduced into the aquarium at the concentration of $0.001 \mathrm{mg} / \mathrm{L}$. Movement of the specimens were continuously observed from the top-view at $0.25 \mathrm{~s}$ intervals before $(10 \mathrm{~h})$ and after $(10 \mathrm{~h})$ the treatments. The analog data captured by the CCTV camera were digitized using a video overlay board (Dooin Electronics Co., Ltd.; OSCARIII ${ }^{\circledR}$ ), and were subsequently sent to the image recognition system (developed by the Artificial Intelligence Laboratory, Department of Electronics Engineering, Pusan National University) to locate the target organism in two-dimension (Fig. 1). The middle point of the body was recorded as the $x, y$ location of the specimens in the observation aquarium. In case the specimens moved, the center of 


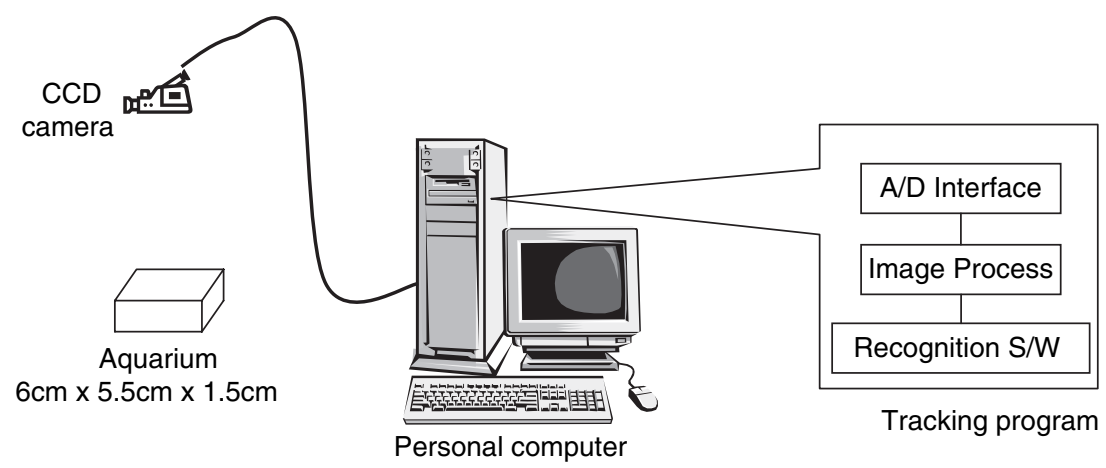

Figure 1: Observation system for automatic recording and detection of movement behaviors of chironomid larvae.

the moving portion of the body was recorded as the position of the specimens. For image processing, stable conditions were maintained for the test specimens in the monitoring system. Disturbances in the observation aquarium were minimized; oxygen and food were not provided to the test specimens during the observation period. Light and temperature conditions were the same to the conditions of the stock population, while the other observation methods were carried out based on Kwak et al. [8] and Park et al. [30].

\subsection{Fractal dimension}

The overall feature of the images on the movement tracks of the specimens was extracted by fractal dimension. The dimension of the movement tracks was addressed as a number through the box counting method. Whilst the topological dimension of a line is always 1 and that of a surface always 2 , the fractal dimension may be any real number between 1 and 2 , since observation was carried out in an aquarium two-dimensionally. The fractal dimension $D_{\mathrm{f}}$ is expressed in the number of boxes, $N(r)$, with scale $r$ covering data points $(x, y)$ (location of the specimens recorded in $0.25 \mathrm{~s}$ interval) [25]:

$$
N(r) \sim r^{D_{\mathrm{f}}} .
$$

$N(r)$ could be plotted versus box size $r$ using a log-log scale. The slope of the log-log plot indicates the fractal dimension $D_{\mathrm{f}}$.

The whole data during the observation time were divided into 1-h segments with the overlapping time for $30 \mathrm{~min}$. For example, if the first segment was initiated from point $0 \mathrm{~h}$ for the first $1 \mathrm{~h}$, the next segment started from point $0.5-1.5 \mathrm{~h}$. Subsequently, the third segment spanned points from 1 to $2 \mathrm{~h}$, and so on. This was continued until the pointer reached $10 \mathrm{~h}$ at the 19 th segment. Time averaged fractal dimension for the segment, $k$, during the total observation period (either before or after the treatments) was defined by:

$$
\overline{D_{\mathrm{f}}}=\frac{1}{T} \sum_{k=1}^{19} D_{\mathrm{f}}(k)
$$

Here, $T(=19)$ is the total number of the image segments. 


\subsection{Self-Organizing Map (SOM)}

While the overall feature of the movement data was computationally evaluated in one parameter, fractal dimension, the movement tracks were further classified by the SOM $[32,33]$ in the segmentexplicit manner (i.e., "segment by segment" training). The SOM, consisting of two layers of input and output (Fig. 2), performs a non-linear projection of data onto a space in low dimension (conventionally two), and provides a patterned map of input data without prior knowledge $[32,33]$. The output layer consists of $M \times N$ computation nodes (i.e., $20 \times 14$ in this study) on a two-dimensional grid. The Euclidian distance between weight vector and input vector was calculated through learning processes. Of output neurons, the best matching neuron, which has the minimum distance, is chosen as the winner. For the best matching neuron and its neighborhood neurons, the new weight vectors are updated as:

$$
w_{i j}(t+1)=w_{i j}(t)+\alpha(t)\left[x(t)-w_{i j}(t)\right],
$$

where $t$ is the iteration time and $\alpha(t)$ is the learning rate. The learning rate accordingly decreases as the system converges. A detailed description regarding application of the SOM to behavioral data was given in $[30,31]$.

Parameters calculated from 1-h segments of the movement tracks were used as input data for the SOM. Based on the experience of test specimens and the results from the previous studies on continuous observation of response behaviors [8,31], the following six parameters of the movement tracks were selected for the test specimens: speed $(\mathrm{mm} / \mathrm{s})$, acceleration $\left(\mathrm{mm} / \mathrm{s}^{2}\right)$, stop number (stop frequency during $1 \mathrm{~h}$ ), maximum length (maximum movement distance observed in the aquarium), locomotory rate $(\mathrm{mm} / \mathrm{s}$, movement distance without stop time), and meander ( $\mathrm{rad} / \mathrm{mm}$, angle change per movement distance).
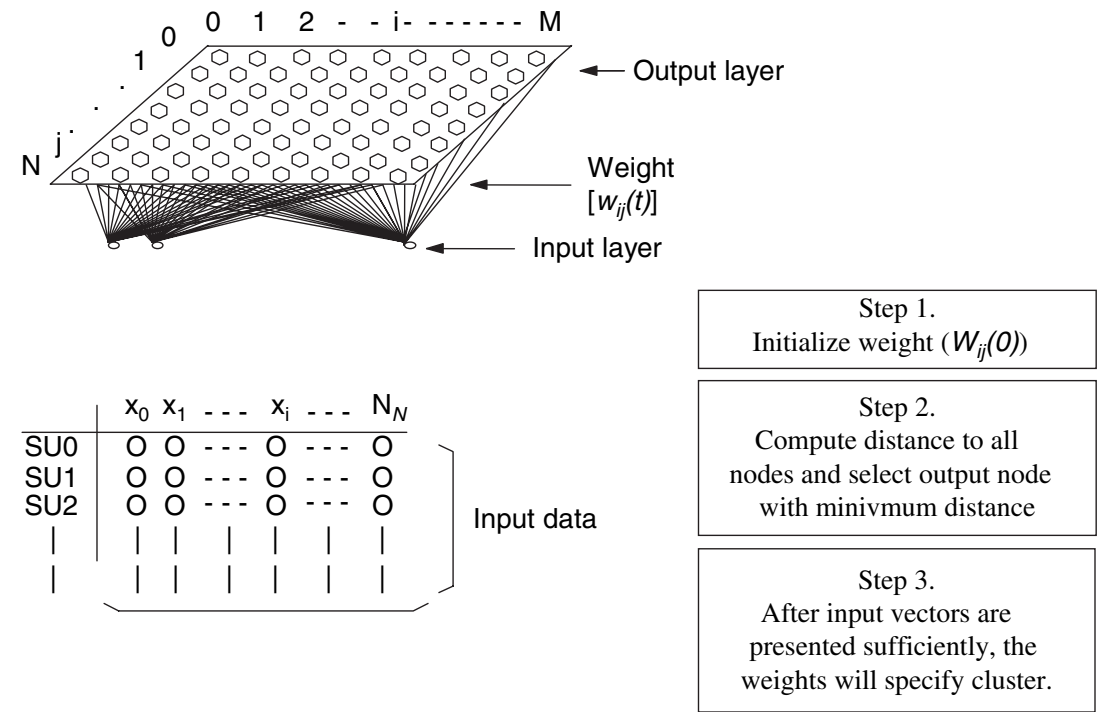

Figure 2: Schematic diagram of the Self-Organizing Map (SOM). 


\section{RESULTS}

\subsection{Fractal dimension}

In general, activity decreased after the treatments of diazinon. Figure 3 shows examples of the time-averaged fractal dimension along with the movement tracks observed in 1-h segments. Before the treatments, the specimens moved actively, and the movement tracks were prevalent on the image of the observation aquarium (Fig. 3(a), (e), (i)). After the treatments, the movement tracks partially occupied the area of observation aquarium (Fig. 3(c), (g), (k)). During exposure to diazinon, intermittent shaking patterns in small scale were observed.

The slope of the log-log scale was almost linear and fractal dimensions were in the range of 1.781.84 before the treatments (Fig. 3(b), (f), (j)), while the slope decreased in the range of 1.60-1.68 after the treatments (Fig. 3 (d), (h), (1)). For all the tested 10 specimens, fractal dimensions consistently decreased (Fig. 4). Based on the paired $t$-test (two tails), significant difference was observed before and after the treatments $(t=7.607, P<0.001)$. This demonstrated that the data structure of the movement tracks was affected by the treatments of diazinon.

\subsection{Self-organizing map (SOM)}

After revealing difference in fractal dimensions before and after the treatments, the different types of the movement tracks were identified using SOM. A dendrogram of the hierarchical cluster analysis was used with the Ward's linkage method based on Euclidean distance in the MATLAB environment (The MathWorks, 1998) (Fig. 5). First, the segments of the movement tracks were broadly separated before and after the treatments. The movement tracks before the treatments occupied the top area

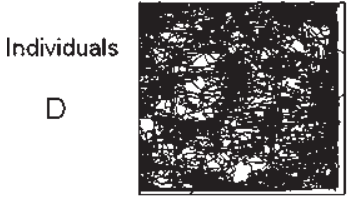

(a)

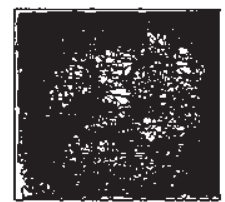

(e)

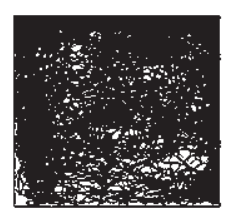

(i)

Movement track

(before treatment)
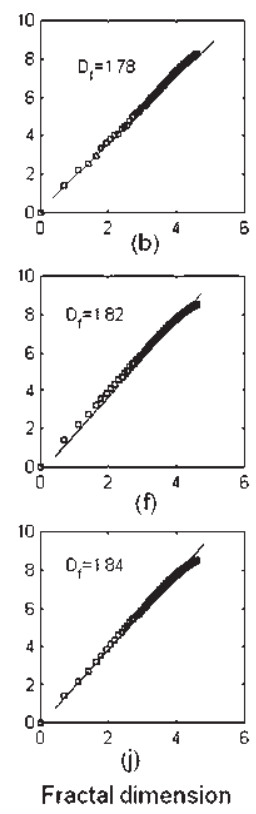

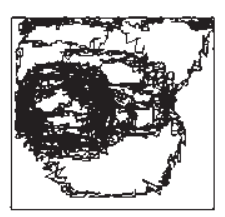

(c)

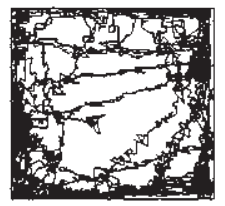

(g)

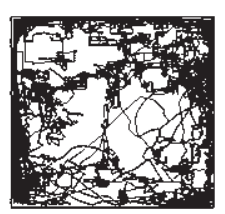

(k)

Movement track
(aftej treatment)
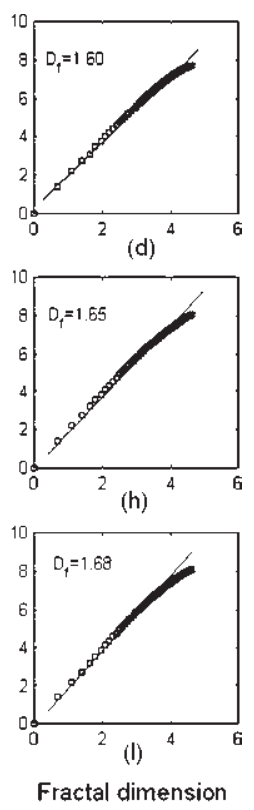

Figure 3: Fractal dimensions measured and the movement tracks $(1 \mathrm{~h})$ of chironomid larvae before and after the treatments. 


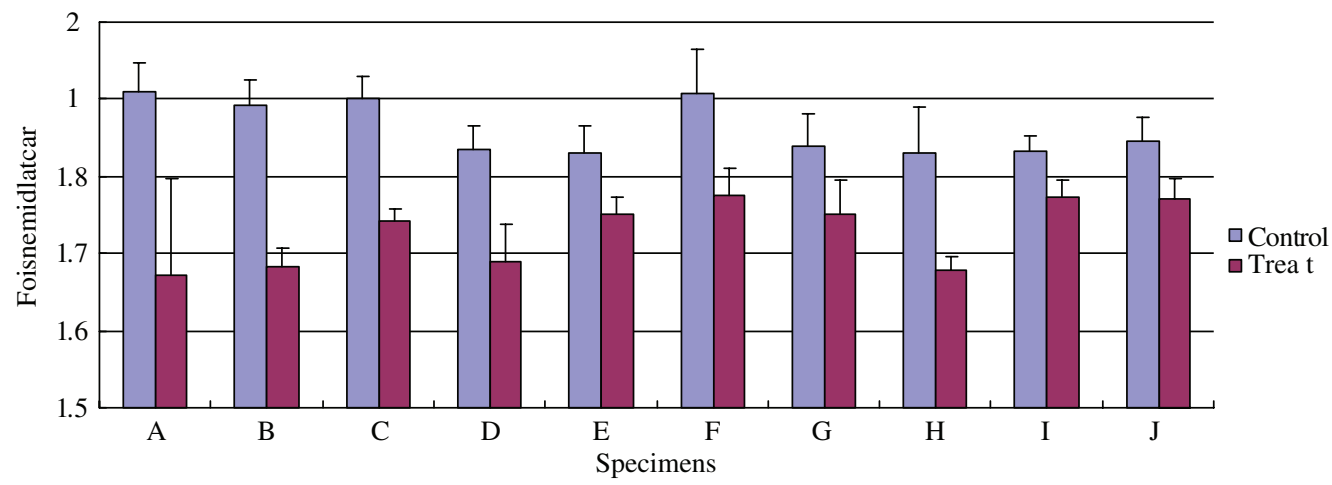

Figure 4: Changes in fractal dimension (average) in different specimens before and after the treatments.

(cluster 1), while the movement tracks after the treatments were placed in the bottom area (Cluster 2). The mapping by SOM confirmed the difference observed in fractal dimension before and after the treatments (Fig. 4), and indicated that variation residing in the movement data was accordingly projected onto the trained SOM. The SOM further classified the different movement patterns in the subclusters as shown on the map (Fig. 5). The movement tracks were accordingly visualized by the profiles of the parameters matching the clusters of the SOM (Fig. 6). Speed, acceleration, and stop number presented the diagonal gradients (from upper right to bottom left), while locomotory rate and meander tended to show the vertical gradient. Maximum length was selectively higher at the upper left corner of the map. Within the upper area (cluster 1), the group of subclusters 1-a and 1-b were further divided from subcluster 1-c (Fig. 5). In subclusters 1-a and 1-b, speed, acceleration, and locomotory rate were in the lower range while stop number and meander were relatively in the higher range (Fig. 6). In contrast to the group of subclusters 1-a and 1-b, the activities shown in subclusters 1-c were in the highest range. This matched the actual movement tracks (Fig. 7). The movement tracks shown in subcluster 1-c covered almost the entire area of the observation aquarium during the observation period $(1 \mathrm{~h})$.

Subclusters 1-a and 1-b were further divided according to differences in maximum length, stop number, and meander (Fig. 6). Subcluster 1-a was characteristically high in maximum length and was relatively lower in meander and stop number compared with subcluster 1-b. Subcluster 1-a presented active movement mainly along the boundary area, while the movement for subcluster 1-b occurred more frequently in the center of the aquarium (Fig. 7). It is understandable that the movement tracks belonging to subcluster 1-a showed the highest range in maximum length since the specimens moved around the boundary area of the observation aquarium.

The movement tracks belonging to the bottom area (cluster 2) were also subdivided. The group of subclusters 2-a and 2-b occupied a relatively smaller area in the left bottom corner of the map and were characteristic with the lowest range in stop number (Fig. 6). The group of subclusters 2-a and 2-b was also in accord with lower speed and lower acceleration compared with subcluster 2-c. Subcluster 2-c was deviated from the group of subclusters 2-a and 2-b by matching with the lowest range in locomotory rate. Subcluster 2-c was also in accordance with the higher levels of stop number, meander, speed, and acceleration. Activities for the group of subcluster 2-a and 2-b appeared to slow down, while stop number also decreased to the minimum range (Fig. 6). In contrast, activities were relatively higher along with higher degree of meandering in subcluster 2-c. Subcluster 2-a was 


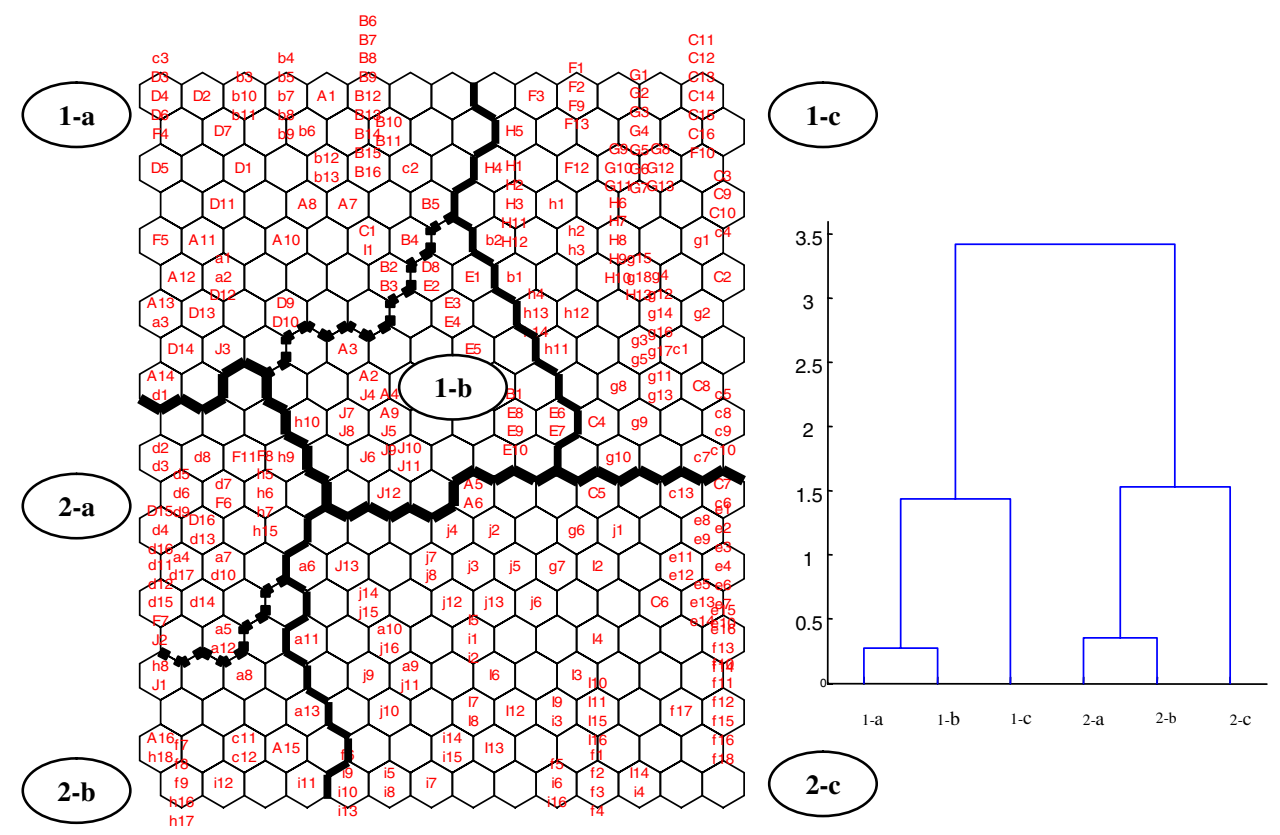

Figure 5: Clustering of the movement tracks by the SOM (left) and cluster distances according to the Ward's linkage method (right). The capital and small alphabets listed on the names of the hexagonal map (left) represent 'before' and 'after' the treatments, respectively.

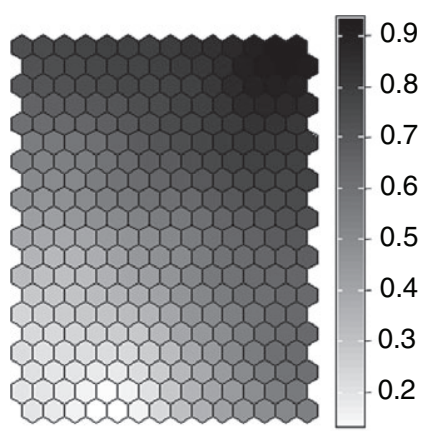

Speed $(\mathrm{mm} / \mathrm{s})$

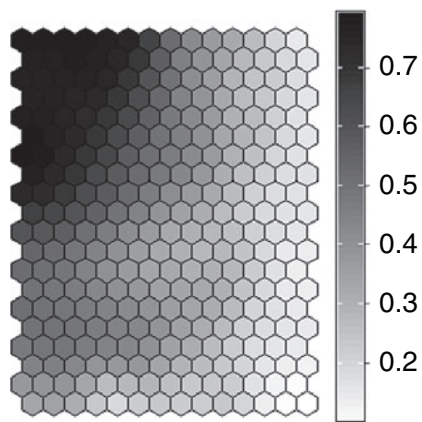

Max_length (mm)

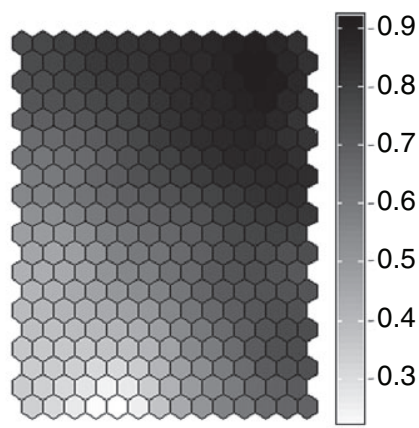

Acceleration $\left(\mathbf{m m} / \mathbf{s}^{2}\right)$

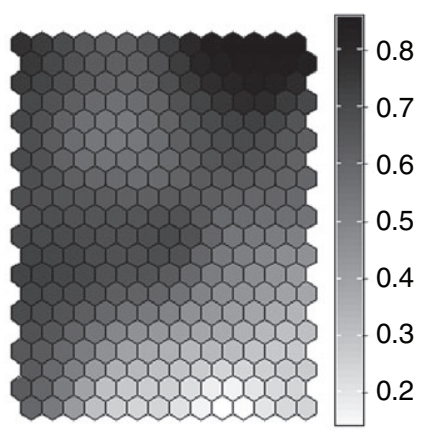

Locomotory_rate $(\mathrm{mm} / \mathrm{s})$

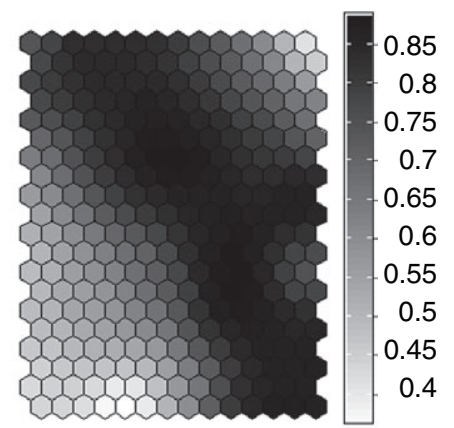

Stop number

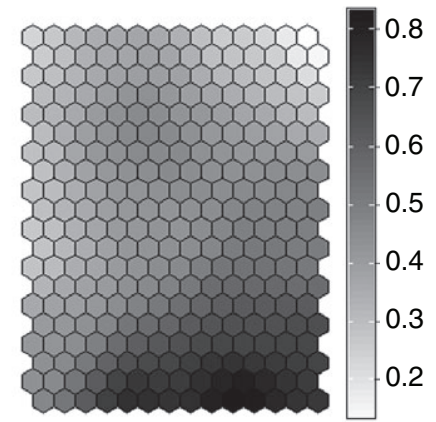

Meander (mm/rad)

Figure 6: Profile of the parameters according to the clusters based on the SOM (Fig. 5). 

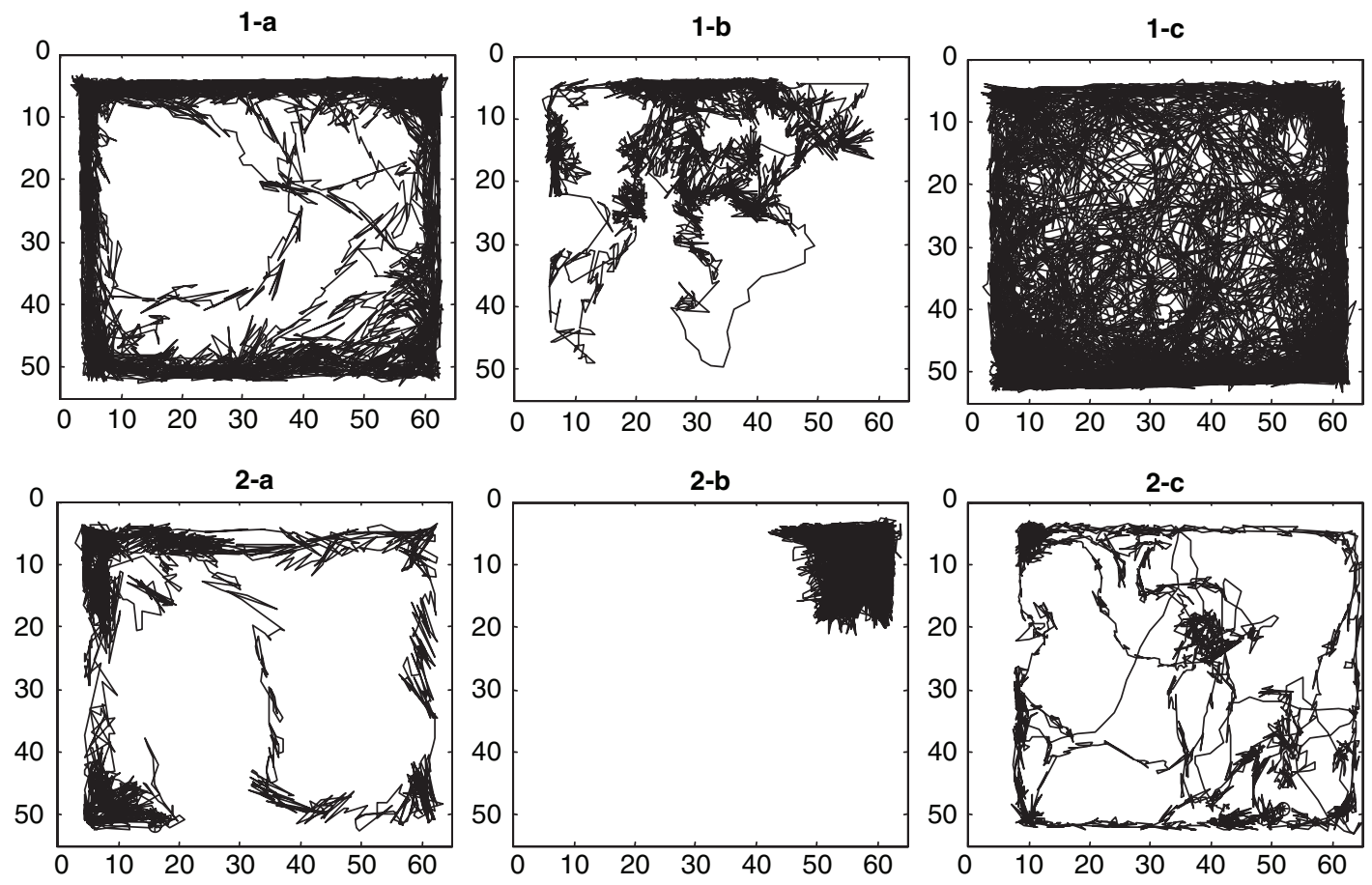

Figure 7: The movement tracks $(1 \mathrm{~h})$ typically observed in different subclusters listed on the SOM (Fig. 5).

further separated from subcluster 2-b by maximum length; shorter for subcluster 2-b and longer for subcluster 2-a (Fig. 6). The parameters shown in subcluster 2-a was characteristic with relatively higher levels of locomotory rate and maximum length, and relatively lower levels of meander.

The movement tracks shown in subcluster 2-c covered a wider range in the aquarium compared with the group of subclusters 2-a and 2-b (Fig. 7). The movement pattern in subcluster 2-c was somewhat similar to the movement tracks shown in subclusters 1-a before the treatments. However, the movement tracks in subcluster 2-c covered less area in the boundary zone and crossed the observation aquarium more frequently. The movement tracks in subcluster 2 -a were similar to the movement tracks in subcluster 1-a in the sense that the specimens in both subclusters 1-a and 2-a stayed at the boundary area for a longer time (Fig. 7). However, activities were generally lower in subcluster 2-a by showing lower levels in speed, acceleration, locomotory rate, and maximum length (Fig. 6).

Statistical difference was accordingly observed between clusters and subclusters (Fig. 8). Speed and acceleration were generally higher in cluster 1, showing the highest range in subclusters 1-c as stated before. Within cluster 2, speed and acceleration were higher in subcluster 2-c than in the other subclusters. Stop numbers showed a similar trend to speed except the case for subcluster 2-c, showing the highest range similar to the level of cluster 1 . Maximum length was distinctively high in cluster 1-a followed by cluster 1-b, while locomotory rate was exceptionally low in subclusters 2-b and 2-c. The opposite trend to locomotory rate was observed in meander with the highest range in the group of subclusters 2-b and 2-c. 

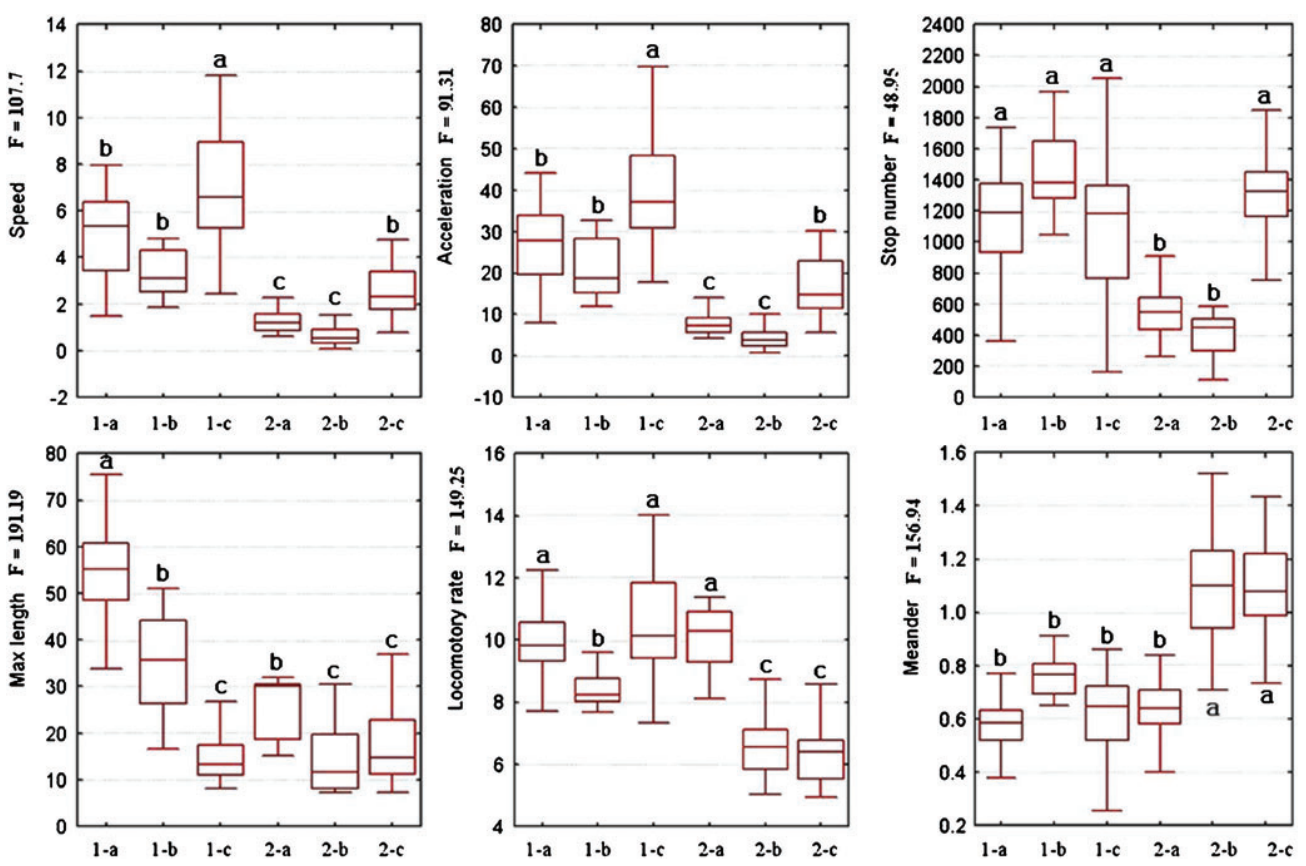

Figure 8: Variation in different parameters of the movement tracks of chironomid larvae according to subclusters listed on the SOM. Statistical significance between the treatments was based on the Tukey test $\left(F_{0.05(1), 6,300}=2.13\right)$. $F$ values (ANOVA) for each parameter were provided on $y$ axes (Fig. 5).

\section{DISCUSSION AND CONCLUSIONS}

In this study, we quantitatively characterized the complex data for behavioral response of the chironomid larvae to chemical treatments. Changes in data structure were revealed by fractal dimension, showing consistent decrease after the treatments (Figs 3-4). These results confirmed previous studies on decrease in fractal dimension in animals such as fish with toxic treatments [31] and ibex with biological disturbances (e.g. parasite and pregnancy) [28].

A similar study has been carried out by Ji et al. [31] by application of fractal dimension and artificial neural network in combination. Ji et al. [31] used the MLP, and the data patterns were initially selected as the templates by experienced observers before training with the MLP. Subsequently, the MLP was used to recognize the trained patterns. After recognition of the MLP, fractal dimension was used to confirm changes in data structure before and after the treatments. In this study, however, we analyzed the movement data without prior knowledge. Initially, changes in data structure were confirmed by calculation of fractal dimensions. Subsequently, the movement tracks were trained with the SOM (Fig. 5) in the segment-explicit manner.

Visualization by the SOM was further useful for characterizing the movement patterns (Figs 6-8). The profiles of parameters in combination were efficient in revealing the overall characterization of the different patterns (Fig. 8). The results led to provision of three types of symptom of the movement behaviors of the test specimens after the treatments of diazinon at low concentration. In addition to the lower levels of speed and acceleration, pattern 1 (subcluster 2-c) showed higher levels of stop number, meander, and lower levels of maximum length and locomotory rate, while pattern 2 
(subcluster 2-b) showed the lowest range in stop number and maximum length. Pattern 3 (subcluster 2-a) was somewhat in between patterns 1 and 2 , showing relatively higher levels of locomotory rate and maximum length, and relatively lower levels of meander.

Among the clusters on the SOM, however, there were some mixed segments between 'before' and 'after' the treatments. In subcluster 1-c, for instance, the movement segments after the treatments were observed, although cluster 1 generally covered the movement segments before the treatments. In this case, however, the mixed segments occurred according to individual specimens in groups. The majority of the movement tracks from individuals $\mathrm{C}, \mathrm{G}$, and $\mathrm{H}$ were included in subcluster 1-c, while the movement segments before and after the treatments from the specimen B occurred in subcluster 1-a. In cluster 2, however, the movement tracks after the treatments were mostly observed. The movement tracks before the treatments in cluster 2 were not observed as frequently as observed in the movement tracks after the treatments shown in cluster 1 . This indicated that the movement patterns observed in cluster 2 were more characteristic in revealing response behaviors of the specimens treated by the chemicals. The mixture of the segments in cluster 1 could be understood from variation of activities. Regarding that subcluster 1-c showed the most active movement patterns (Figs 6-8), some strongly active individuals could still remain in subcluster 1-c after the treatments. This was understandable by considering that concentration applied in this study was at a fairly low level. According to clustering in Fig. 5 (cluster 2), the data obtained in this investigation demonstrated that the changes in movement tracks are inducible at a low dose of diazinon $(0.001 \mathrm{mg} / \mathrm{L})$. Although similar behavioral studies on the larvae of Chironomus riparius treated with diazinon have not been reported, diazinon has been treated at the range of $0.1-10 \mathrm{mg} / \mathrm{L}$ for behavioral study on other animals $[8,30,34,35]$.

In conclusion, complex data of response behaviors of indicator species treated with toxic chemicals could be analyzed by fractal dimension and SOM. Fractal dimensions were consistent in showing lower values after the treatments. SOM was feasible in classifying the movement patterns before and after the treatments in the segment-explicit manner and would be useful for systemically characterizing the symptoms of the affected specimens. The use of fractal dimension and the SOM in combination could be an alternative tool for automatically monitoring toxic substances in environment.

\section{ACKNOWLEDGMENTS}

This article was supported by the Korea Science and Engineering Foundation (KOSEF; Project no. R01-2004-000-11036-0).

\section{REFERENCES}

[1] Lemly, A.D. \& Smith, R.J., A behavioral assay for assessing effects of pollutants of fish chemoreception. Ecotoxicology and Environmental Safety, 11, pp. 210-218, 1986.

[2] Dutta, H., Marcelino, J. \& Richmonds, Ch., Brain acetylcholinesterase activity and optomotor behavior in bluegills, Lepomis macrochirus exposed to different concentrations of diazinon. Arch. Intern. Physiol. Biochem. Biophys., 100, pp. 331-334, 1992.

[3] Blübaum-gronau, E., Hoffmann, M., Spieser, O.H. \& Krebs, F., The 'Koblenz behavioral fish test', an automated biotest based on the video processing system BehaviQuant ${ }^{\circledR}$ for monitoring of water quality, Schrifenreihe des Vereins für Boden-, Wasser- und Lufthygiene, 93, pp. 87-117, 1994.

[4] Lorenz, R., Spieser, O.H. \& Steinberg, C., New ways to ecotoxicology: Quantitative recording of behaviour of fish as toxicity endpoint. Acta hydrochim. Hydrobiol., 23, pp. 197-201, 1995. 
[5] Lee, S.E. \& Lee, S.K., Biological sensors for detecting aquatic toxicity of hazardous chemicals using respiratory and movement responses of carp, Cyprinus capio. J. Korean Soc. Water Quality, 12, pp. 119-126, 1996.

[6] Moore, A. \& Waring, C.P., Sublethal effects of the pesticide diazinon on olfactory function in mature male Atlantic salmon parr. J. Fish Biol., 48, pp. 758-775, 1996.

[7] Gray, M.A., Teather, K.L. \& Metcalfe, C.D., Reproductive success and behavior of Japanese Medaka (Oryzias latipes) exposed to 4-tert-octylphenol. Environmental Toxicology and Chemistry, 18, pp. 2587-2594, 1999.

[8] Kwak, I.-S., Chon, T.S., Kang, H.M., Chung, N.I., Kim, J.S., Koh, S.C., Lee, S.K. \& Kim, Y.S., Pattern recognition of the movement tracks of medaka (Oryzias latipes) in response to sub-lethal treatments of an insecticide by using artificial neural networks. Environmental Pollution, 120, pp. 671-681, 2002.

[9] Oshima, Y., Kang, I.J., Kobayashi, M., Nakayama, K., Imada, N. \& Honjo, T., Suppression of sexual behavior in male Japanese medaka (Oryzias latipes) exposed to $17 \beta$-stradiol. Chemosphere, 50, pp. 429-436, 2003.

[10] St-Amand, L., Gagnon, R., Packard, T.T. \& Savenkoff, C., Effects of inorganic mercury on the respiration and the swimming activity of shrimp larvae, Panaslus borealis. Comparative Biochem. and Physiol. Part C, 112, pp. 33-43, 1999.

[11] Abgrall, P., Rangeley, R.W., Burridge, L.E. \& Lawton, P., Sublethal effects of azamethiphos on shelter use by juvenile lobsters (Homarus americanus). Aquaculture, 181, pp. 1-10, 2000.

[12] Roast, S.D., Widdows, J. \& Jones, M.B., Disruption of swimming in the hyperbenthic mysid Neomysis integer (Peracarida: Mysidacea) by the organophosphate pesticide chlorpyrifos. Aquatic Toxicology, 47, pp. 227-241, 2000.

[13] Burridge, L.E., Haya, K., Waddy, S.L. \& Wade, J., 2000, 'The lethality of anti-sea lice formulations Salmosan ${ }^{\circledR}$ (Azamethiphos) and Excis ${ }^{\circledR}$ (Cypermethrin) to stage IV and adult lobsters (Homarus americanus) during repeated short-term exposures. Aquaculture, 182, pp. 27-35, 2000.

[14] Ibrahim, W.L.F., Furu, P., Ibrahim, A.M. \& Christensen, N.Ø., Effect of the organophosphorous insecticide, chlorpyrifos (Dursban), on growth, fecundity and mortality of Biomphalaria alexandrina and on the production of Schistosoma mansoni cercariae in the snail. Journal of Helminthology, 66, pp. 79-88, 1992.

[15] Gerhardt, A., Janssens de Bistoven, L. \& Soares, A. M. V. M., Effect of acid mine drainage and acidity on the activity of Choroterpes picteti (Ephemeroptera: Leptophlebiidae). Environmental contamination and toxicology, 48, pp. 450-458, 2005.

[16] Alt, W., Correlation analysis of two-dimensional locomotion paths. Lecture Notes in Biomathematics, eds W. Alt \& G. Hoffman, Springer-Verlag: Berlin, pp. 254-268, 1990.

[17] Scharstein, H., Paths of carabid beetles walking in the absence of orienting stimuli and the time structure of their motor output. Lecture Notes in Biomathematics, eds W. Alt \& G. Hoffman, Springer-Verlag: Berlin, pp. 269-277, 1990.

[18] Tchernichovski, O., Benjamini, Y. \& Golani, I., The dynamics of long-term exploration in the rat. Part I. A phase-plane analysis of the relationship between location and velocity. Biol. Cybernetics, 78, pp. 423-432, 1998.

[19] Tchernichovski, O. \& Benjamini, Y., The dynamics of long-term exploration in rat. Part II. An analytical model of the kinematic structure of rat exploratory behavior. Biol. Cybernetics, $\mathbf{7 8}$, pp. 433-440, 1998.

[20] Drai, A., Benjamini, Y. \& Golani, I., Statistical discrimination of natural modes of motion in rat exploratory behavior. Journal Neuroscience Methods, 96, pp. 119-131, 2000. 
[21] Gerhardt, A., Carlsson, A., Ressemann, C. \& Stich, K.P., New online biomonitoring system for Gammarus pulex (L.) (Crustacea): in situ test below a copper effluent in south Sweden. Evnrionmental Science Technology, 32, pp. 150-156, 1998.

[22] Gerhardt, A., Janssens de Bistoven, L. \& Penders, E., Quality control of drinking water from the river Rhine with multispecies freshwater biomonitor. Aquatic Ecosystem Health \& Management, 6, pp. 159-166, 2003.

[23] Tourtellot, M.K., Collins, R.D. \& Bell, W.J., The problem of move length and turn definition in analysis of orientation data. Journal of Theoretical Biology, 150, pp. 287-297, 1991.

[24] Barnsley, M.F., Fractals Everywhere, Morgan Kaufmann: San Diego, 1993.

[25] Johnson, A.R., Milne, B.T. \& Wiens, J.A., Diffusion in fractal landscapes: simulations and experimental studies of tenebrionid beetle movements. Ecology, 73, pp. 1968-1983, 1992.

[26] Wiens, J.A., Crist, T.O. \& Milne, B.T., On quantifying insect movement. Environmental Entomology, 22, pp. 709-715, 1993.

[27] Wiens, J.A., Crist, T.O., With, K.A. \& Milne, B.T., Fractal patterns of insect movement in microlandscape mosaics. Ecology, 79, pp. 663-666, 1995.

[28] Alados, C.L., Escos, J.M. \& Emlen, J.M., Fractal structure of sequential behaviour patterns: an indicator of stress. Animal Behaviour, 51, pp. 437-443, 1996.

[29] Chon, T.-S., Park, Y.-S., Park, J.Y., Choi, S.-Y., Kim, K.T. \& Cho, E.C., Implementation of computational methods to pattern recognition of movement behavior of Blattella germanica (Blattaria: Blattellidae) treated with $\mathrm{Ca}^{2+}$ signal inducing chemicals. Appl. Entomol. Zool., 39, pp. 79-96, 2004.

[30] Park, Y.-S., Chung, N.-I., Choi, K.-H. Cha, E.Y., Lee, S.-K. \& Chon, T.-S., Computational characterization of behavioral response of medaka (Oryzias latipes) treated with diazinon.Aquatic Toxicology, 71, pp. 215-228, 2005.

[31] Ji, C.W., Lee, S.H., Kwak, I.-S., Cha, E.Y., Lee, S.-K. \& Chon, T.-S., Computational analysis of movement behaviors of medaka (Oryzias latipes) after the treatments of copper by using fractal dimension and artificial neural networks, Environmental Toxicology, eds A.G. Kungolos, C.A. Brebbia, C.P. Samaras \& V. Popov, WIT Press: Southampton and Mykonos, pp. 93-107, 2006.

[32] Kohonen, T., Self-organized formation of topologically correct feature maps. Biol. Cybern., 43 pp. 59-69, 1982.

[33] Kohonen, T., Self-Organizing Maps, Springer: Berlin, 2001.

[34] Köprücü, S.Ş., Köpröcü, K., Ural, M.Ş., İspir, Ü. \& Pala, M., Acute toxicity of organophosphorous pesticide diazinon and its effects on behavior and some hematological parameters of fingerling European catfish (Silurus glanis L.). Pesticide Biochemistry and Physiology, 86, pp. 99-105, 2006.

[35] Sun, X.-B., Cui, F-Y., Zhang, J.-S., Xu, F. \& Liu, L.-J., Inactivation of chironomid larvae with chlorine dioxide. Journal of Hazardous Materials, in press, 2006. 